Article

\title{
Applying Population Viability Analysis to Inform Genetic Rescue That Preserves Locally Unique Genetic Variation in a Critically Endangered Mammal
}

\author{
Joseph P. Zilko ${ }^{1, *(\mathbb{D})}$, Dan Harley ${ }^{2}$, Alexandra Pavlova ${ }^{1}\left(\mathbb{D}\right.$ and Paul Sunnucks ${ }^{1}(\mathbb{D}$ \\ 1 School of Biological Sciences, Monash University, Clayton, VIC 3800, Australia; \\ alexandra.pavlova@monash.edu (A.P.); paul.sunnucks@monash.edu (P.S.) \\ 2 Zoos Victoria, Parkville, VIC 3052, Australia; dharley@zoo.org.au \\ * Correspondence: joseph.zilko@monash.edu
}

check for updates

Citation: Zilko, J.P.; Harley, D.; Pavlova, A.; Sunnucks, P. Applying Population Viability Analysis to Inform Genetic Rescue That Preserves Locally Unique Genetic Variation in a Critically Endangered Mammal. Diversity 2021, 13, 382. https:// doi.org/10.3390/d13080382

Academic Editors: Kym Ottewell and Margaret Byrne

Received: 30 June 2021

Accepted: 12 August 2021

Published: 16 August 2021

Publisher's Note: MDPI stays neutral with regard to jurisdictional claims in published maps and institutional affiliations.

Copyright: (c) 2021 by the authors. Licensee MDPI, Basel, Switzerland. This article is an open access article distributed under the terms and conditions of the Creative Commons Attribution (CC BY) license (https:// creativecommons.org/licenses/by/ $4.0 /)$.

\begin{abstract}
Genetic rescue can reduce the extinction risk of inbred populations, but it has the poorly understood risk of 'genetic swamping' - the replacement of the distinctive variation of the target population. We applied population viability analysis (PVA) to identify translocation rates into the inbred lowland population of Leadbeater's possum from an outbred highland population that would alleviate inbreeding depression and rapidly reach a target population size $(N)$ while maximising the retention of locally unique neutral genetic variation. Using genomic kinship coefficients to model inbreeding in Vortex, we simulated genetic rescue scenarios that included gene pool mixing with genetically diverse highland possums and increased the $N$ from 35 to 110 within ten years. The PVA predicted that the last remaining population of lowland Leadbeater's possum will be extinct within 23 years without genetic rescue, and that the carrying capacity at its current range is insufficient to enable recovery, even with genetic rescue. Supplementation rates that rapidly increased population size resulted in higher retention (as opposed to complete loss) of local alleles through alleviation of genetic drift but reduced the frequency of locally unique alleles. Ongoing gene flow and a higher $N$ will facilitate natural selection. Accordingly, we recommend founding a new population of lowland possums in a high-quality habitat, where population growth and natural gene exchange with highland populations are possible. We also recommend ensuring gene flow into the population through natural dispersal and/or frequent translocations of highland individuals. Genetic rescue should be implemented within an adaptive management framework, with post-translocation monitoring data incorporated into the models to make updated predictions.
\end{abstract}

Keywords: genetic swamping; genetic rescue; locally unique alleles; translocation; population viability analysis; population genetics; marsupial; adaptive potential; Leadbeater's possum; Gymnobelideus leadbeateri

\section{Introduction}

Many threatened species are restricted to small and isolated populations, which places them at elevated risk of inbred matings and loss of beneficial genetic variation by genetic drift [1,2]. Small and isolated populations of diploid species that usually outbreed nearly always exhibit reduced fitness as a consequence of inbreeding (i.e., inbreeding depression) [3]. Usually concurrent with inbreeding depression, the loss of beneficial variation and the accumulation of harmful variation further reduce the population mean fitness [4]. Together with the loss of genetic variation that would otherwise facilitate adaptation to future environmental conditions, these issues reduce population viability [1,5]. If inbred populations remain isolated, extinction becomes increasingly likely due to the 'extinction vortex' effect, wherein a shrinking population size is reinforced by increasing inbreeding depression and fitness loss [6,7].

Translocations of individuals from other populations can increase genetic diversity of isolated populations and elevate population fitness by reducing inbreeding depression 
and alleviating the harmful effects of beneficial variation being lost from the population (i.e., causing genetic rescue), thus increasing the persistence and adaptive capacity of populations $[2,8,9]$. Such genetic management is a highly effective conservation tool; when appropriate sources of genetic augmentation are used, outcrossing increases fitness in the vast majority of inbred populations that usually outbreed, and an enhanced ability to adapt can greatly increase population persistence in changing environments [10].

Despite its demonstrated effectiveness, genetic rescue has not been widely implemented, partly owing to concerns about 'genetic swamping' and a consequent loss of local adaptation and distinctiveness [11-13]. Genetic swamping can be defined as the replacement of a population's locally adaptive genetic variation with that of immigrants [13]. This definition may be extended to include apparently neutral alleles within populations because distinguishing adaptive from non-adaptive variation can be extremely challenging, and such variation can contribute to future adaptive potential [14]. An extreme example of genetic swamping occurs in some hybridising species, in which hybrid vigour (heterosis) causes the extirpation of the original locally adapted population and their alleles [15]. A subtler form of genetic swamping occurs when locally unique genetic variation is replaced during the immigration of conspecifics [16]. Concerns about genetic swamping tend to promote conservation strategies that maintain genetic distinctiveness of isolated populations rather than evaluating alternative management options in a risk-benefit framework, but a different strategy might have a better outcome for the stated conservation goals [17]. On one hand, species-wide genetic variation could be lost if translocation strategies cause the replacement of locally unique alleles, potentially reducing the adaptive potential and increasing extinction risk [13,18-20]. Conversely, the genetic distinctiveness of small and isolated populations is often an artefact of genetic stochasticity rather than local adaptation, and if 'uniqueness' reflects harmful variation that would have been removed by selection in larger and/or more connected populations, maintaining the distinctiveness of small populations can even exacerbate extinction risk [21].

Effective population sizes $\left(N_{e}\right)>100$ are needed to limit the mean inbreeding coefficient (the probability that any two alleles are identical by descent) to $F<0.1$ over five generations [10]. Smaller and more inbred populations need high supplementation rates to reverse inbreeding, which could elevate the risk of swamping [1]. Nevertheless, alleviating inbreeding depression while avoiding the replacement of the local gene pool may be achievable by appropriately managing gene flow during genetic rescue. Even under considerable gene flow during intensive genetic rescue, selection can maintain locally adaptive genetic variation in the face of potential swamping [22]. However, selection is generally weaker in small populations because genetic drift has a greater influence on allele frequencies [23]. Preserving genome-wide variation, including variation that is currently neutral, also enhances future adaptive potential $[14,24,25]$. Thus, it is of interest to estimate how much locally unique genome-wide variation will persist in a population under genetic rescue, especially if candidate populations for genetic rescue are small. Accordingly, poor understanding of swamping during genetic rescue is a significant knowledge gap [13].

Population demographic and genetic models that account for inbreeding depression and the genetic contribution of outbred immigrants could be useful for designing genetic rescue regimes that help retain locally unique alleles. Forward-based computer simulations can project the changes in the genetic profile of populations under different demographic and population genetic scenarios [26]. Such simulations have been used to estimate the impacts of inbreeding depression and predict changes in the viability of inbred populations after genetic rescue [14,27]. PVAs can be used to predict how much of the genetic variation from a source population can be retained when founding a new population or supplementing an existing population [28-30]. However, the preservation of alleles unique to a local population that is supplemented with unrelated individuals has been less scrutinised, and fitness disparities between outbred immigrants and inbred locals can cause the replacement of local variation [31]. Incorporating inbreeding and inbreeding depression into models accounts for the reduced contribution to the gene pool of inbred locals relative to immi- 
grants. Models that consider locally unique variation and the lower fitness of inbred locals relative to immigrants could therefore be useful for designing genetic rescue programs that improve population viability without replacing the local gene pool.

Leadbeater's possum (Gymnobelideus leadbeateri) is a critically endangered Australian marsupial and a strong candidate for genetic rescue. Genetic rescue of this species could be informed by population viability analysis (PVA) modelling the effect of various translocation scenarios on the retention of unique alleles when the recipient gene pool has reduced fitness due to severe inbreeding. The species has suffered extensive loss and deterioration of habitat caused by vegetation clearing, bushfires, and logging, and has a highly restricted distribution (Figure 1) within a $70 \times 95 \mathrm{~km}$ area within the Central Highlands of Victoria, Australia, with a neighbouring lowland isolate [32,33]. The single population of Leadbeater's possum outside of the Central Highlands is localised entirely within a narrow $6 \mathrm{~km}$ stretch of lowland swamp forest habitat in the Yellingbo Nature Conservation Reserve [34]. The Yellingbo population is genetically distinct from highland populations and is thought to represent the sole remnant of a previously widespread evolutionary lineage adapted to lowland swamp $[32,35,36]$. The geographic isolation of the Yellingbo population makes contemporary gene flow with other populations highly unlikely [36]. This population had greatly reduced genetic diversity compared to all conspecific populations and exhibited substantial inbreeding depression for survival to sexual maturity, longevity, probability of breeding during lifetime, and lifetime reproductive output [37]. Inbreeding depression likely contributed to the decline from 120 individuals in 1997 to 35 individuals in 2017. Heterozygosity concurrently declined by $12 \%$, putatively limiting the capacity of the population to adapt to environmental change and prevent the expression of deleterious variation [37]. Furthermore, suitable habitat for Leadbeater's possum has declined in extent and quality, mainly because of habitat succession and altered hydrology [32]. Based on monotonic decline in population size, presence of inbreeding depression, and deteriorating habitat condition, the extinction of the Yellingbo population is highly likely without intervention $[37,38]$. Translocation of lowland Yellingbo individuals to high-quality habitat beyond Yellingbo and outcrossing with genetically diverse highland animals is planned to prevent the extinction of this population. Sourcing migrants for genetic rescue of the lowland population from highland populations is the only available option, which has been assessed to have a low risk of outbreeding depression [37]. Although genetic variation unique to the lowland population at Yellingbo has been characterised with microsatellite markers and short mitochondrial sequences [36], it was not assessed with genome-wide markers, which should better approximate functional and standing variation [24,25]. Doing so would be an important step in planning translocation strategies that do not replace the local gene pool.

We aimed to design a translocation regime of lowland and highland Leadbeater's possums into new habitat that would rapidly increase population size, demographic stability, and genetic diversity, while providing for the retention of unique lowland alleles. We predicted the level of retention of alleles unique to the lowland population, incorporating inbreeding depression to reflect the potential fitness advantage of translocated outbred individuals and their descendants over inbred locals. We used genome-wide SNP markers to detect genomic variation unique to the lowland population, which was then monitored across simulated scenarios. We also used genome-wide SNPs to model inbreeding and genetic diversity within simulated populations. The following objectives were targeted:

(1) Predict the trajectory of the lowland population at Yellingbo without genetic rescue;

(2) Determine whether genetic rescue can retain genetic variation unique to the lowland population;

(3) Assess whether more rapid genetic rescue presents a greater risk of uniquely lowland variation being replaced.

Our results will inform genetic rescue of the last extant population of the lowland lineage of this critically endangered species. More generally, our approach for design- 
ing genetic rescue regimes to retain locally unique variation should be useful for other conservation programs.

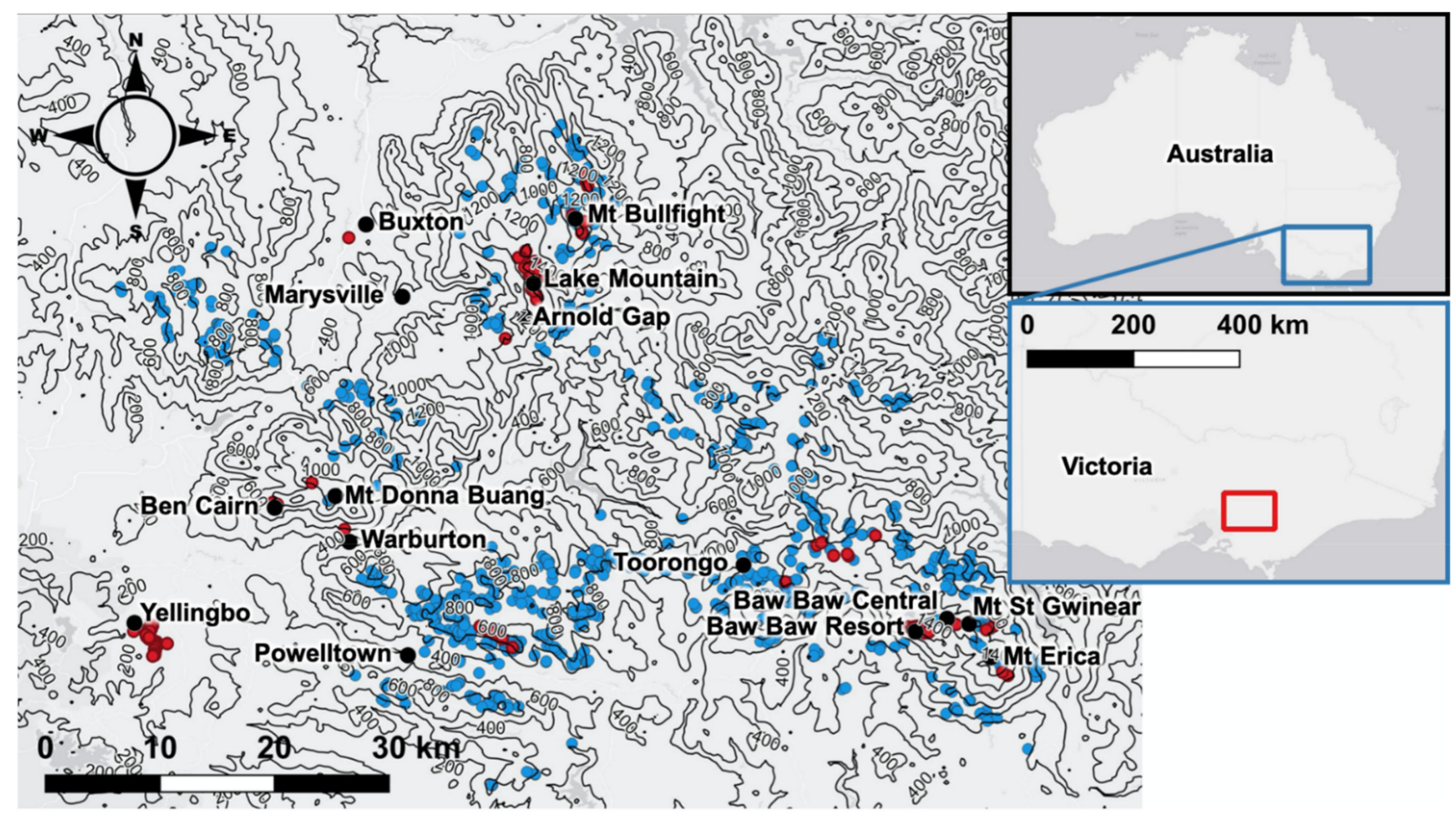

Figure 1. Map of sampling locations for genetic samples used in this study (red dots). The blue dots are observations of the species from 2010-2020, indicating the contemporary range. The extent of the sampling area is indicated by the red rectangle on the inset. Contour lines indicate elevation above sea level in metres.

\section{Materials and Methods}

\subsection{Study System and Sampling}

The lowland population at Yellingbo was extensively surveyed from 1997-2019, with mark-recapture data available for $>70 \%$ of the population in any given year $[37,39]$. These data provided estimates of key demographic parameters, such as mortality and reproduction rates (see below). Genetic samples $(n=304)$ were collected from Yellingbo during surveys from 1997 to 2001 and 2011 to 2019 [36,37]. A further 216 individuals were genetically sampled throughout the species' range in the Central Highlands (Figure 1), against which to estimate the locally unique variation at Yellingbo. These included 113 individuals from Lake Mountain-the thoroughly sampled population used to represent allele frequencies of a donor highland population in simulations. For most samples, a small $\left(\sim 2 \mathrm{~mm}^{2}\right)$ tissue biopsy of skin and cartilage was taken from the outer pinna of the ear and stored in ethanol prior to extraction. Skin samples were also taken from animals found dead or from the pelts of preserved museum specimens.

\subsection{Defining Conservation Targets}

Conservation programs guided by PVAs require clearly defined criteria of success [40]. We defined a strategy as successful if it resulted in a population of at least 110 individuals. This target was chosen to recreate apparent demographic stability observed at Yellingbo in the 1990s before the rapid deterioration of habitat conditions and concurrent population decline. The limited availability of lowland habitat also means that founded populations must necessarily be $<120$ individuals in size, with translocations maintaining population size and genetic diversity. This target represents a first (and urgent) milestone to prevent the extinction of the lowland population; however, further population expansion would 
be required thereafter. We assumed that genetic rescue would begin with 20 Yellingbo founders, which represents $57 \%$ of the population in 2019 .

\subsection{General Assumptions of the PVA Model}

All PVAs were performed using the software package Vortex v.10.3.5.0 [41], an individual-based Monte Carlo simulation program that models the impact of deterministic and stochastic forces on animal populations. Demographic, environmental, and genetic processes were incorporated by inputting parameter estimates for mortality and fecundity, along with environmental variability. For a range of scenarios (Table 1), the program tracked individuals from birth to death, with reproduction and mortality occurring at pre-defined probabilities, derived from actual population data (below). Environmental catastrophes were not included in the model. Due to the uncertainty of environmental effects on fitness, environmental effects were minimised by setting the standard deviation for mortality and reproduction parameters to $10 \%$ of their mean value (Table 2). This was possible only for the parameters modelled using mean and standard deviation values.

Table 1. Scenarios simulated in Vortex. Initial lowland population size $(N)$, carrying capacity $(K)$, whether or not the starting population was made inbred by providing a kinship matrix (Inbred), whether inbreeding depression was modelled, and whether estimated or adjusted values for juvenile ( $0-1$ y) mortality were used is indicated. Supplementation rate is the number of individuals supplemented (by translocation) into the population per year. Supplementation duration is the number of years the population was supplemented. Simulation duration is the number of years simulations were run. Source is the population that animals were sourced from for supplementation.

\begin{tabular}{|c|c|c|c|c|c|c|c|c|c|}
\hline Scenario & $\mathbf{N}$ & K & Inbred & $\begin{array}{l}\text { Inbreeding } \\
\text { Depression }\end{array}$ & $\begin{array}{l}\text { Juvenile } \\
\text { Mortality }\end{array}$ & $\begin{array}{c}\text { Supplementation } \\
\text { Rate }\end{array}$ & $\begin{array}{c}\text { Supplementation } \\
\text { Duration (Years) }\end{array}$ & $\begin{array}{c}\text { Simulation } \\
\text { Duration (Years) }\end{array}$ & Source \\
\hline \multirow{2}{*}{ 1990s Condition } & \multirow{2}{*}{110} & 120 & No & Yes and no & Estimated & 0 & NA & 50 & NA \\
\hline & & 1000 & No & Yes and no & Estimated & 0 & NA & 50 & NA \\
\hline \multirow{2}{*}{2019 Trajectory } & \multirow{2}{*}{35} & 40 & Yes & Yes & $6 \%$ & 0 & NA & 50 & NA \\
\hline & & 120 & Yes & Yes & $6 \%$ & 0 & NA & 50 & NA \\
\hline $\begin{array}{l}\text { Demographic } \\
\text { Rescue }\end{array}$ & 20 & 1000 & Yes & Yes & $6 \%$ & $2 ; 4 ; 6 ; 8 ; 10$ & 50 & 50 & Lowland \\
\hline Genetic Rescue & 20 & 1000 & Yes & Yes & $6 \%$ & $2 ; 4 ; 6 ; 8 ; 10$ & 50 & 50 & Highland \\
\hline $\begin{array}{c}\text { Genetic } \\
\text { Swamping Test }\end{array}$ & 20 & 1000 & Yes & Yes & $6 \%$ & $2 ; 4 ; 6 ; 8 ; 10$ & 50 & $10 ; 20 ; 30 ; 40 ; 50$ & Highland \\
\hline
\end{tabular}

Table 2. Biological parameters used in Vortex simulations. The study that provided each parameter estimate is given.

\begin{tabular}{|c|c|c|}
\hline Parameter & Value (SD) & Source \\
\hline Diploid lethal equivalents & 6.29 & [42] \\
\hline Percent inbreeding due to lethal recessives & 50 & [43] \\
\hline Environmental variation correlation between reproduction and survival & 0 & Not used \\
\hline Environmental variation correlation among populations & 0 & Not used \\
\hline Age producing first offspring (years) & 2 & [39] \\
\hline Maximum lifespan (years) & 9 & [37] \\
\hline Maximum litters per year & $2 *$ & [39] \\
\hline Maximum progeny per litter & 2 & [39] \\
\hline Maximum age of reproduction (years) & 9 & [37] \\
\hline Sex ratio at birth & 01:01 & [39] \\
\hline Percent adult females breeding & $66.6(6.7)$ & [39] \\
\hline \multicolumn{3}{|l|}{ Distribution of litters per year } \\
\hline 0 litter & 0 & {$[44]$} \\
\hline
\end{tabular}


Table 2. Cont.

\begin{tabular}{|c|c|c|}
\hline Parameter & Value (SD) & Source \\
\hline 1 litter & 7 & [44] \\
\hline 2 litter & 93 & {$[44]$} \\
\hline \multicolumn{3}{|l|}{ Distribution of offspring per litter } \\
\hline 1 offspring & 45 & [39] \\
\hline 2 offspring & 55 & [39] \\
\hline \multicolumn{3}{|l|}{ Mortality } \\
\hline 0-1 years (males) & $42(4.2)+$ & [44] \\
\hline $0-1$ years (females) & $41(4.1)+$ & {$[44]$} \\
\hline 1-2 years (males) & $17(1.7)$ & [44] \\
\hline 1-2 years (females) & $22(2.2)$ & [44] \\
\hline Annual >2 years (males) & $29(2.9)$ & [37] \\
\hline Annual >2 years (females) & $29(2.9)$ & [37] \\
\hline Percent males in breeding pool & 100 & \\
\hline
\end{tabular}

Genetic data were incorporated into all but one analysis (1990s Condition being the exception) as the input allele frequencies and pairwise kinships among individuals (detailed methods below). Alleles were assumed to be neutral and randomly assorting. Vortex uses pairwise kinships to calculate inbreeding coefficients for individuals and their offspring. Inbreeding depression was included in some models as a reduced survival probability in the first year of life (the default in Vortex). Inbreeding depression is caused by an increased expression of deleterious recessive alleles and a loss of heterozygote advantage with increasing autozygosity [45]. Lethal equivalents represent recessive alleles that compromise fitness when homozygous in an individual, with a single homozygous lethal equivalent imposing a fitness cost equivalent to the death of an individual [3]. Decreases in individual survival probability are determined by the number of lethal equivalents, the relative contribution of lethal recessive alleles and the heterozygote advantage to inbreeding depression, and the individual inbreeding coefficient. The default value of 6.29 diploid lethal equivalents for first year survival was used. This value was used in preference to estimates of lethal equivalents in the lowland Leadbeater's possum population obtained in a previous study because these estimates were found to have wide confidence intervals and varied between different inbreeding coefficients [37]. Vortex randomly assigns lethal equivalents to each founder at the beginning of simulations. In Vortex, homozygosity for any lethal recessive always results in mortality in the first year of life. The relative contribution of lethal and sub-lethal recessive alleles to inbreeding depression is not well understood, and therefore, we specified $50 \%$ inbreeding depression due to lethal recessive alleles, a classic empirical estimate from fruit flies [43]. The reduction in first year survival caused by reduced heterozygote advantage is calculated by applying an exponential equation that includes the inbreeding coefficient of an individual.

\subsection{Genotyping}

DNA was extracted by salting-out [46] or using the DNeasy Blood and Tissue Kit following Qiagen's (the manufacturer) instructions. The preparation of reduced-representation genome libraries, sequencing, and the discovery of genome-wide single nucleotide polymorphism loci (SNPs) was carried out using the DArTseq complexity reduction methodology of Diversity Arrays Technology [47] and described in a previous study [37]. This generated 13368 SNP loci that were then filtered for downstream analysis using the $R$ package dartR [48], as explained below. The function gl.sexlinkage was used to detect 
putatively sex-linked loci, which were removed from the dataset. Loci containing more than one SNP were thinned to retain the SNP with the highest polymorphism information. Locus reproducibility was determined during marker discovery from technical replicates representing $25 \%$ of all data, and loci with average reproducibility $<100 \%$ were removed from the dataset to minimise error rates.

Three genetic datasets were then created by sub-setting the data by sampling location and applying different quality-filtering criteria in dart $R$ to meet the requirements of each downstream analysis. The first dataset of 4218 loci, created by retaining individuals from all sampling locations and removing loci with a call rate of $<85 \%$, was used to detect alleles unique to the lowland population at Yellingbo. The second dataset of 1066 loci, created by retaining only the Lake Mountain and Yellingbo populations and removing loci with a call rate of $<100 \%$, was used during simulations to represent genetic variation at Yellingbo and a thoroughly sampled representative highland population. The third dataset of 1076 loci, created by retaining only Yellingbo individuals and removing loci with a call rate of $<99 \%$, was used to calculate pairwise kinships at Yellingbo.

\subsection{Detecting Alleles Unique to the Lowland Population}

Alleles occurring only within the lowland population at Yellingbo (locally unique alleles) were detected by comparing the allele frequencies of all highland individuals pooled together with allele frequencies of lowland individuals sampled at Yellingbo from 1997-2019. The package poppr [49] was used to report unique alleles and counts of each locally unique allele in the population. Loci with locally unique alleles found as a single copy in only one Yellingbo individual were removed, as these could represent genotyping errors or rare deleterious mutations. Allele frequencies calculated for loci with locally unique alleles for Yellingbo individuals sampled in 2011-2019 were used in genetic rescue simulations.

\subsection{Pairwise Kinship Estimation}

Inbreeding among individuals in the population was estimated using pairwise kinships, which measure the proportion of the genome that is identical by shared ancestry between pairs of individuals [50]. Kinships calculated using genetic markers often better represent genomic similarity than do pedigree-derived estimates [51]. Pairwise kinships were calculated with the beta.dosage function in the $R$ package hierfstat [52] using 1076 loci and all Yellingbo genotypes from 1997 to 2019. This kinship estimator has a mean of zero for the focal population, with negative values representing below-average kinship and positive values representing above-average kinship [53]. Vortex requires positive kinship values that represent the probability that any two homologous alleles drawn from a pair of individuals are identical by descent [41]. Therefore, we used the transformation described previously [54] to convert calculated kinships to proportions, with the resulting pairwise kinship value for the least-related pair equal to zero. Kinship values for the 2019 population were incorporated into simulations as a matrix. By default, Vortex regards non-genotyped individuals as completely outbred and unrelated, which is unrealistic. Accordingly, five non-genotyped individuals were represented in the kinship matrix with pairwise kinship values set to the mean calculated for the 2019 individuals.

\subsection{Survival and Reproduction Parameters Used in Vortex Models}

The Vortex models were populated with realistic parameter values (Table 2) using published estimates of reproduction and mortality at different life stages [39]. The age classes for which that study calculated mortality differed from those required by Vortex. As such, mortality from 0 to 1 years was calculated as the sum of total litter loss (9\%) for both sexes, plus mortality for each sex from when juveniles first emerged from the pouch to 12 months old. The mean annual adult ( $>2$ years old) mortality rate was estimated by averaging the annual estimates from 1996 to 1999. 
Based on the observation of a relatively stable Yellingbo population at 110 individuals in the 1990s, simulations using realistic demographic parameters were expected to yield a demographically stable population-that is, one that did not change in size by more than $10 \%$ over 50 years in the absence of inbreeding depression or environmental catastrophes (see scenario 1990s Condition below). However, simulations using published mortality estimates resulted in a rapidly growing population (Supplementary Figure S1). To achieve demographic stability, we increased the mortality rates for individuals aged 0 to 1 years by $6 \%$ for both sexes to $42 \%$ in males and $41 \%$ in females. Actual mortality at $<3$ months old was likely underestimated in the empirical studies because the initial number of young present in the pouch at birth (one or two) could not readily be established, preventing a reliable estimation of partial litter loss [39]. Accordingly, increasing mortality at age $0-1$ years by $6 \%$ to achieve demographic stability was deemed a realistic adjustment.

\subsection{Sensitivity Analysis}

The sensitivity of PVAs to input parameter estimates was investigated using perturbation analysis-using simulations of scenario 1990s Condition (with no inbreeding depression) while increasing or decreasing a single input parameter by $10 \%$ relative to its baseline value (Table 2 ). The average growth rate $(r$ ) was calculated from 200 replicate runs. The growth rate was used to assess model sensitivity because other parameters, such as survival probability and population size, have theoretical limits of zero and/or one, which limits their usefulness for estimating effect size. The relative sensitivity of the model to each parameter was calculated with the following formula: $\left(r_{+}-r_{-}\right) /\left(0.2 \times r_{0}\right)$, where $r_{+}$is the mean $r$ with the parameter increased by $10 \%, r_{-}$is the mean $r$ with the parameter decreased by $10 \%, r_{0}$ is the mean $r$ using the baseline parameter value, and 0.2 is equal to the perturbation in the parameter value. These estimates indicated whether certain parameters had a disproportionate effect on models, which can make predictions less reliable if the parameter estimates are inaccurate.

\subsection{Simulated Scenarios}

Five general scenarios were tested, which are outlined in Table 1 and described in detail below. For all scenarios except 1990s Condition, the starting population was specified using a studbook file (a list of founder individuals and their characteristics in Vortex) that included the age and sex of each individual at Yellingbo in 2019. Relationships among individuals were not provided in the studbook file. Instead, a kinship matrix was also provided for all scenarios except 1990s Condition to estimate individual inbreeding. For scenarios in which the lowland population was supplemented with highland individuals, the population started with the same set of 20 (10 male and 10 female) Yellingbo founders that were randomly sampled from the studbook containing 35 Yellingbo individuals in 2019. Translocated animals had the same mortality and reproduction rates as the recipient population once added to the population (not including inbreeding depression effects), and all were assumed to survive translocation. For each simulated scenario, the extinction probability was estimated as the proportion of replicate runs that went extinct, with extinction defined as only individuals of one sex remaining; this definition was used in all subsequent simulations. Mean and standard deviation for output parameter estimates were generated using 200 replicate iterations for each scenario. Increasing the number of replicates above 200 did not decrease the standard error of estimates. Simulation outputs included observed heterozygosity, population size $(N)$, growth rate across all years $(r)$, and time to reach $N=110$.

(i) 1990s Condition: The effect of limited carrying capacity and inbreeding on population size at Yellingbo

The influence of the limited carrying capacity and inbreeding on the lowland population size at Yellingbo since the 1990s was determined. A population of 110 individuals with all pairwise kinships equal to zero was simulated to represent the population in the 1990 s, varying the carrying capacity to $K=120$ and $K=1000$. These scenarios were run 
using actual juvenile mortality estimates rather than those increased by $6 \%$ to achieve a demographically stable baseline in other scenarios. Simulations at these carrying capacities were run with and without inbreeding depression.

(ii) 2019 Trajectory: The forward projection of the population size of lowland possums at Yellingbo without genetic rescue

The time until extinction of the lowland population at Yellingbo without genetic rescue was estimated with simulations, starting with 35 lowland individuals alive in 2019 in the absence of supplementation and specifying pair-wise kinships, including inbreeding depression and as actual juvenile mortality rates (as for the 1990 Condition above). These simulations were carried out using two different carrying capacities; $K=40$, representing the currently degraded habitat conditions, and $K=120$, representing higher quality habitat present in the 1990s.

(iii) Demographic Rescue: The effect of numerical population reinforcement on population growth

Scenarios were run to separate the effect of supplementation on population growth (i.e., demographic rescue) from the effect of genetic rescue. The effect of supplementation on population size was estimated by introducing migrants from a donor population genetically similar to the lowland population. The donor population was made genetically similar to that of Yellingbo by setting non-zero kinships between Yellingbo and donor individuals. Kinship values were set at the beginning of each run by randomly sampling from a Poisson distribution with a mean equal to the average pairwise kinship between individuals at Yellingbo (mean $=0.5$, the kinship matrix is provided in the Supporting Data). This was also done for pairwise kinships between individuals within the donor population to make it similarly inbred to Yellingbo. Input allele frequencies for this donor population were identical to those of the lowland population.

(iv) Genetic Rescue: The supplementation of a new lowland population with highland possums

All genetic rescue scenarios were carried out assuming that a new population would be founded using 20 lowland individuals relocated from Yellingbo. To represent the founding of a new population in high-quality habitat, a carrying capacity of 1000 was specified in genetic rescue scenarios. Scenarios were run to determine the minimum supplementation rate required to establish a demographically stable ( $N$ changing by $<10 \%$ over 50 years) population of at least 110 individuals, starting with 20 lowland founders (Table 1). This target size was chosen to recreate the apparent demographic stability observed at Yellingbo in the 1990s and early 2000s [39]. The number of supplemented individuals increased by increments of two (one of each sex) to maintain equal sex ratios during simulations.

For genetic rescue scenarios, a highland population was created using allele frequencies calculated for the Lake Mountain population. These highland individuals were specified as unrelated to each other, and unrelated to lowland individuals, by setting pairwise kinships to zero. This enabled the simulation of genetic rescue by introducing outbred individuals. The size of the highland population was set to 1000 individuals with the mean and standard deviation set to 1 for survival at all life stages to ensure that the population remained sufficiently large for translocation and maintained genetic diversity for the duration of simulations. All other parameters were the same as those used for the lowland population at Yellingbo.

Genetic rescue effects were measured as changes in the mean observed heterozygosity and the mean population size from the first to the last year of simulation, the mean growth rate for all years $(r)$, and the mean time to reach $N=110$. Changes in genetic diversity in the rescued population were modelled by specifying the observed allele frequencies for 1066 loci for the highland population and lowland individuals alive in 2019, and using these to calculate heterozygosity for each year of simulation.

(v) Genetic Swamping Test: Determining whether a higher supplementation rate risks the replacement of locally unique alleles 
Vortex outputs summary statistics for loci only at the end of simulations. Thus, allele frequencies and allele retention probabilities were estimated in a time series; genetic rescue scenarios were repeated with simulations that ran for 10, 20, 30, and 40 years. These values were compared between different supplementation rates to determine whether higher rates of gene flow reduced the retention of locally unique alleles. Whether unique lowland allele retention probabilities and frequencies differed significantly among supplementation rates was determined using aligned rank transformation analysis of variance (ART-ANOVA) in the $R$ package ARTool v.0.10.7 [55]. This test is suitable for non-parametric data with paired observations with categorical predictors. Generalised linear models were fit with ARTool with the mean retention probability or frequency as the response, the translocation rate as a predictor, and with data paired by specifying a random intercept for each allele.

Only 23 loci with locally unique lowland alleles were detected (see Section 3.4). Because such a small number of locally unique alleles would make it difficult to predict the effect of the starting allele frequency on retention probability, we used 230 alleles with frequencies replicating those of the 23 observed unique alleles. Frequencies for these unique alleles were set to zero in the highland population. All alleles were assumed to be independently assorting and selectively neutral. The retention probability for each unique lowland allele was given by the proportion of replicate runs in which it did not go extinct.

\section{Results}

\subsection{Sensitivity Analysis}

Perturbation analysis indicated that no single input parameter had a greatly disproportionate effect on the population growth rate (Table 3). Annual adult mortality (2+ years) had the greatest relative impact on the population growth rate, closely followed by the litter size (average number of individuals per litter). Point values for $r$ at each parameter value are given in Supplementary Figure S4.

Table 3. Sensitivity analysis output. The relative sensitivity of PVAs to different input parameters based on perturbation analysis.

\begin{tabular}{ll}
\hline Input Parameter & Sensitivity Index \\
\hline $0-1$ year annual mortality & -0.29 \\
\hline $1-2$ year annual mortality & -0.11 \\
\hline 2+ year annual mortality & -0.44 \\
\hline Proportion of females breeding & 0.35 \\
\hline Brood size & 0.39 \\
\hline
\end{tabular}

\subsection{Trajectory of the Lowland Population without Genetic Management}

In the absence of inbreeding depression, simulations using demographic rates estimated in the 1990s showed that population growth was limited by the carrying capacity (Figure 2). A large carrying capacity of 1000 resulted in positive growth $(r=0.02, \mathrm{SD}=0.08)$ for the 50-year simulated timeframe. Limiting the carrying capacity to a more realistic value of 120 resulted in population decline $(r=-0.013, \mathrm{SD}=0.10)$ for the duration of the simulations. When inbreeding depression was incorporated into the models that assumed that individuals in the starting population are unrelated $(\mathrm{MK}=0)$, the population declined regardless of whether the carrying capacity was $1000(r=-0.028, \mathrm{SD}=0.12)$ or $120(r=-0.039, \mathrm{SD}=0.15)$, although in the former case, it initially increased for half of the simulation period (Figure 2). 

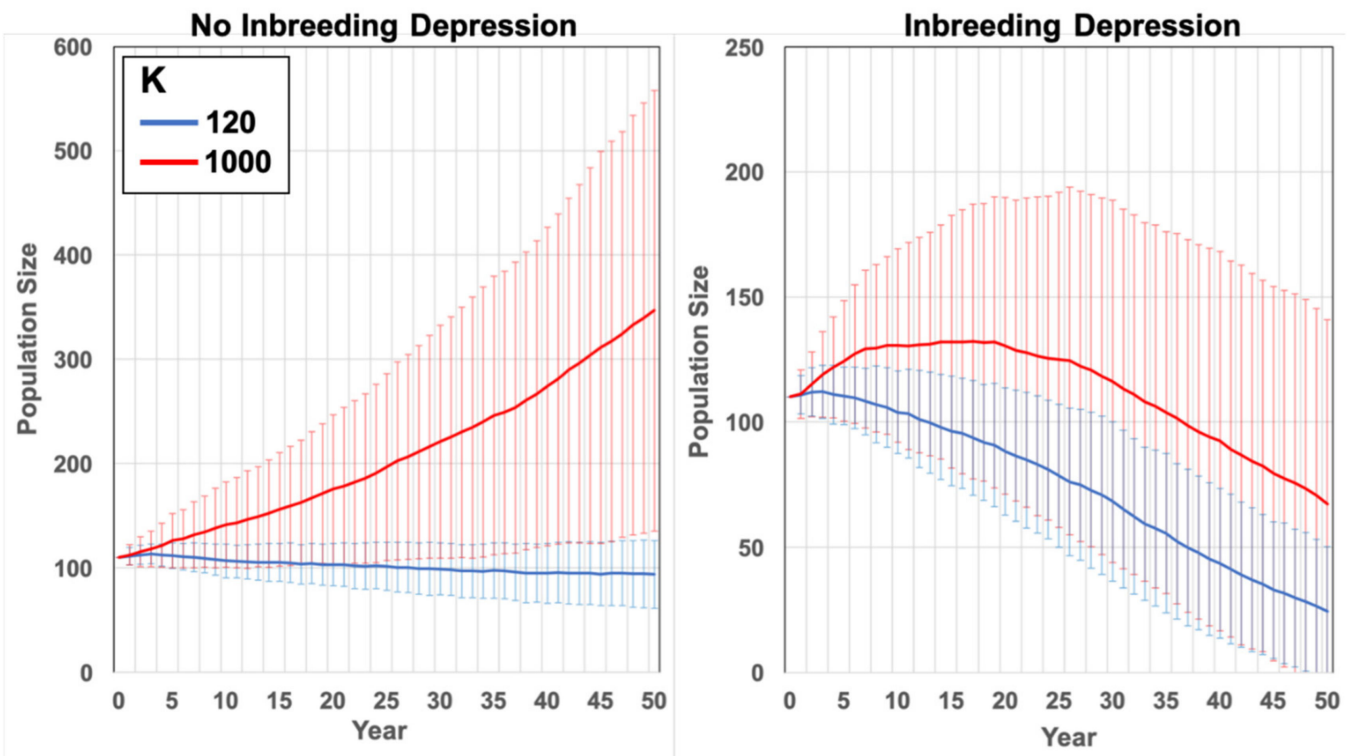

Figure 2. Simulated change in the mean population size \pm 1 SD for 200 replicate simulations using mortality estimates for lowland individuals at Yellingbo in the 1990s, with inbreeding depression either excluded or included in models. Simulations were carried out with the carrying capacity $(K)$ set to 120 to reflect the limited habitat availability in the reserve, and 1000 to determine whether greater habitat availability could yield a demographically stable population without inbreeding depression. Simulation settings are detailed in Table 1 (1990s Condition).

Simulations of the Yellingbo population from 2019 onwards without genetic rescue and with the level of inbreeding informed by genetic estimates (kinship matrix and 2019 allele frequencies) resulted in extinction probabilities of 50\% within 11 years, and 100\% within 23 years (Figure 3$)$. The mean time to extinction was 11.7 years $(\mathrm{SD}=3.4$ ) with a growth rate $(r)$ of $-0.20(\mathrm{SD}=0.24)$ regardless of the carrying capacity $(K=40$ or $K=120)$. This suggests that the lowland population will likely be extinct within two decades without genetic rescue, even if habitat quality is improved.
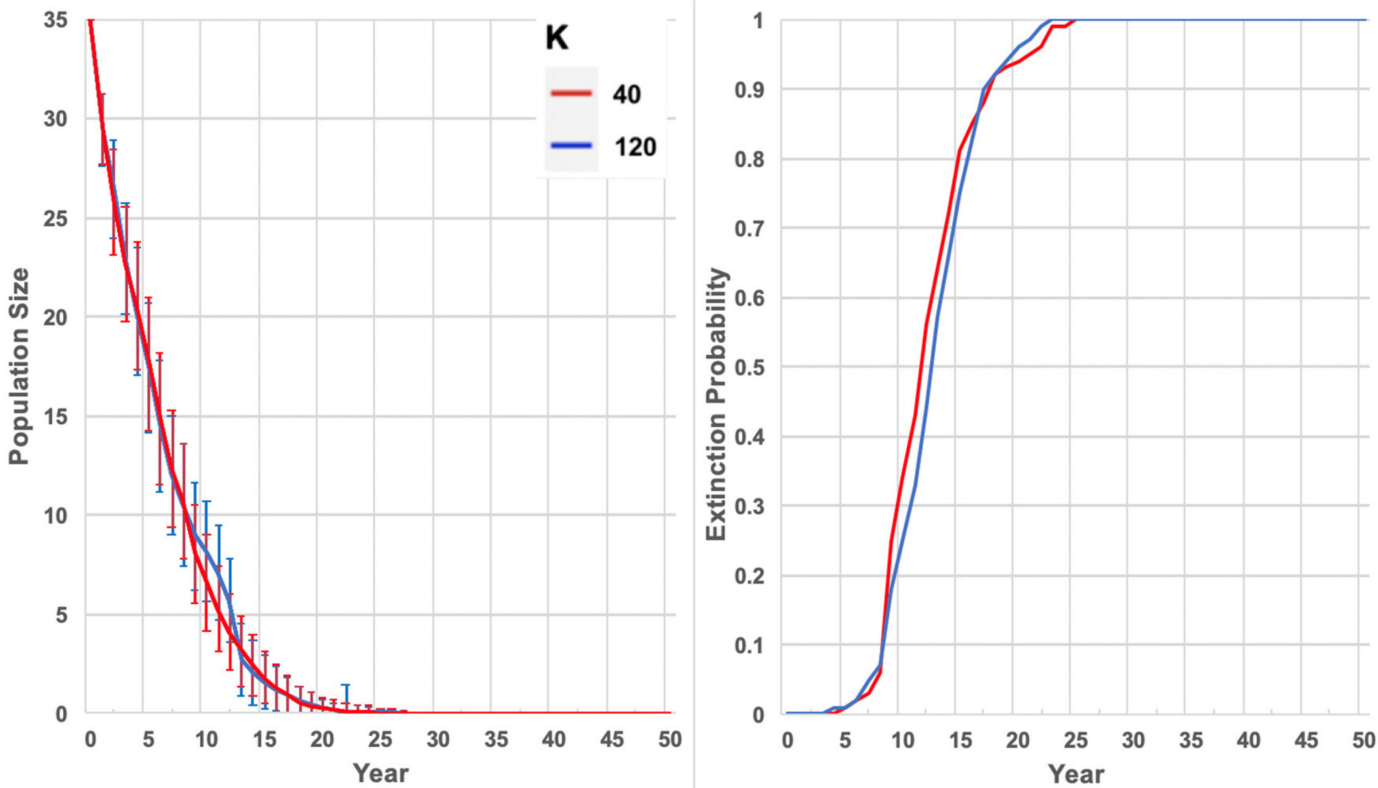

Figure 3. The mean population size $( \pm 1 \mathrm{SD})$ and probability of extinction averaged across 200 replicate simulations, beginning with 35 individuals in Yellingbo with inbreeding depression effects included in the models. The extinction probability is given as the proportion of simulations that had gone extinct by each year, with extinction defined as only individuals of one sex remaining. Simulation settings are given in Table 1 (2019 Trajectory). 


\subsection{Effect of Genetic and Demographic Rescue on Lowland Population Recovery}

Mean population size at the end of simulations of genetic rescue scenarios increased with the supplementation rate (Figure 4). Supplementation with 10 highland individuals per year was the only scenario to reach the target size within 10 years, taking an average of $8.27(\mathrm{SD}=2.0)$ years with a growth rate of $r=0.07(\mathrm{SD}=0.1)$. This was closely followed by supplementation with 8 individuals per year, which took 11 years to reach the target size with a growth rate of $r=0.06(\mathrm{SD}=0.09)$.
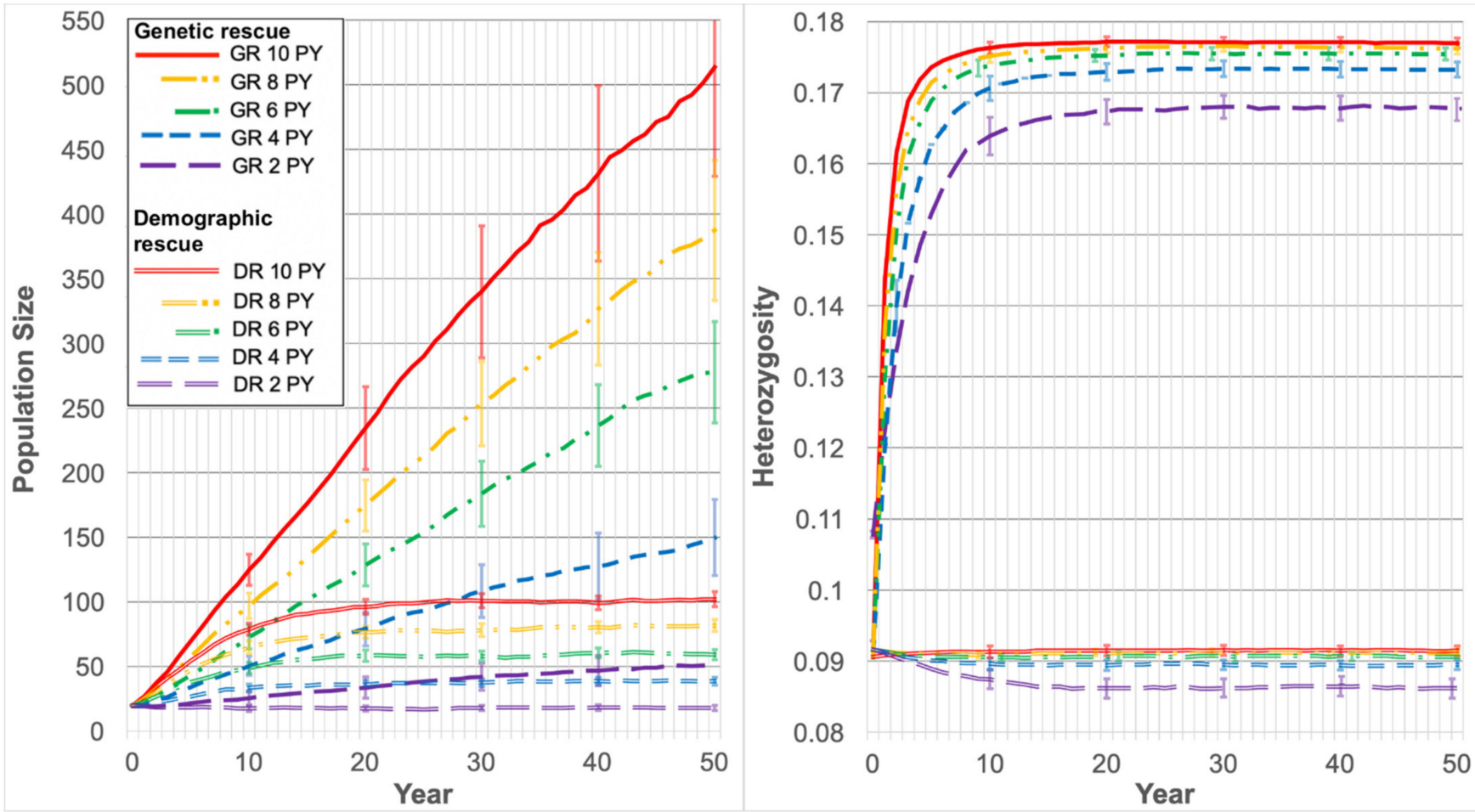

Figure 4. The mean population size $\pm 1 \mathrm{SD}$ and heterozygosity $\pm 1 \mathrm{SD}$ for simulations translocating two to ten individuals per year into the inbred lowland population, beginning with 20 lowland founders. Inbreeding depression as included in the models and $\mathrm{K}=1000$. For genetic rescue (GR) scenarios, unrelated individuals were translocated from a simulated highland population. For demographic rescue (DR) scenarios, individuals were introduced with the same allele frequencies and mean pairwise kinships as individuals in the lowland population in 2019. Simulation settings are given in Table 1 (Genetic Rescue and Demographic Rescue).

Alleviation of inbreeding had an effect distinguishable from merely increasing population size; demographic rescue scenarios (supplementation from the genetically Yellingbolike population) failed to produce positive population growth in simulations regardless of the supplementation rate (Figure 4). The highest mean population size for the demographic rescue scenarios was 102 individuals and was reached after 49 years at a supplementation rate of 10 individuals per year. Varying the contribution of lethal recessive alleles from $0-75 \%$ did not substantially alter the simulation outcomes, although increasing the contribution of lethal alleles unrealistically to $100 \%$ resulted in much higher population viability and larger genetic rescue effects (Supplementary Figure S2).

Mean heterozygosity across replicate runs also increased with the supplementation rate for genetic rescue. The highest mean heterozygosity reached in simulations was $0.177(\mathrm{SD}=0.001)$, at a supplementation rate of 10 individuals per year. This was the highest value possible, given the genetic diversity of the highland population (heterozygosity $=0.18$ ). Maximum heterozygosity was reached sooner with higher rates of gene flow. For genetic rescue, all translocation rates reached at least $90 \%$ of the source population's heterozygosity within ten years. As with the population size, the increase in genetic diversity from genetic rescue was distinguishable from that of demographic rescue. The highest heterozygosity for any 
demographic rescue scenario was 0.092 , which was the highest possible, matching the genetic diversity at Yellingbo.

\subsection{Locally Unique Alleles in the Lowland Population at Yellingbo}

Out of 4218 loci examined, 23 loci $(0.55 \%)$ were found to harbour alleles that occurred only in the lowland population. This excluded 70 alleles that occurred in the heterozygous state in a single individual and were removed; including these alleles would increase the proportion of loci with unique lowland alleles to $2.2 \%$. A large proportion of the locally unique alleles were very rare; out of 23 locally unique alleles, $5(22 \%)$, were found at frequencies below $1 \%$ in the lowland population (Figure 5a). When all Yellingbo samples from 2011-2019 were considered together, the highest recorded frequency for any unique allele was $57.7 \%$. The highest frequency of any unique allele in 2019 was $19.9 \%$. Of the 23 alleles detected from 2011-2019, 13 (57\%) were not detected in 2019 (noting that 14\% of individuals were not genotyped in this year).
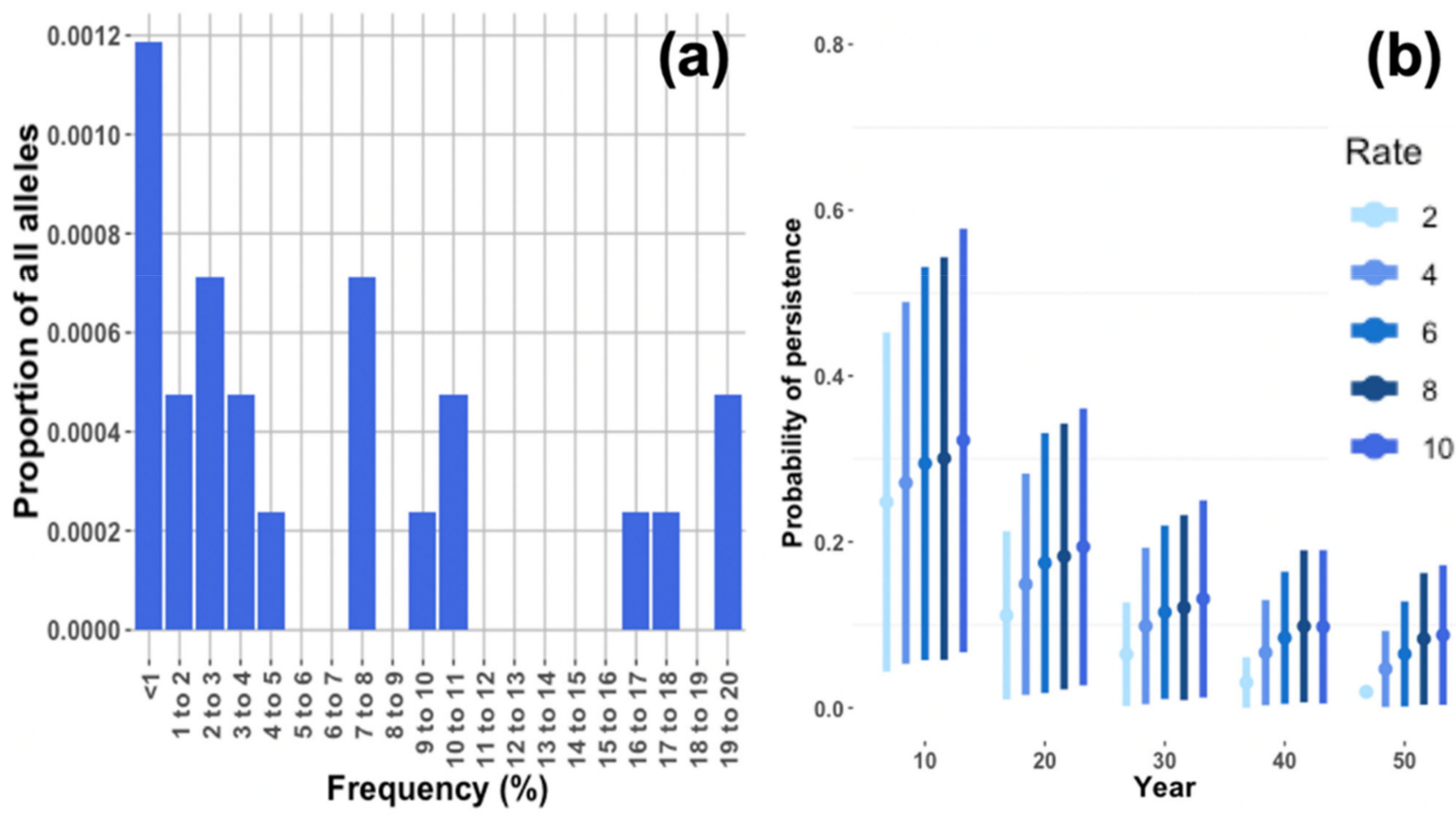

Figure 5. (a) Frequency distribution of locally unique alleles in the lowland population in 2019. Counts of alleles are expressed as a proportion of all alleles in the dataset $(n=4218)$. Frequencies of locally unique alleles from 2011 to 2019 are shown in Supplementary Figure S3. (b) Mean \pm 1 SD retention probability for locally unique alleles of any initial frequency during 50 years of simulated genetic rescue. Simulations began with 20 Yellingbo individuals, supplemented with different numbers of highland individuals per year (Rate). Probability and frequency values were output every ten years, with values calculated from 200 replicate runs of each scenario. A carrying capacity of 1000 was specified.

\subsection{Risk of Decreased Retention of Locally Unique Alleles from Genetic Rescue}

The overall mean retention probability for locally unique alleles was $0.29(\mathrm{SD}=0.01)$ at 10 years of genetic rescue, declining to $0.06(\mathrm{SD}=0.07)$ at 50 years (Figure $5 b)$. When considered separately, the retention probability for alleles with starting frequencies $>10 \%$ (Figure 6a) during genetic rescue were somewhat higher, with the highest mean retention probability after 10 years of genetic rescue being $0.68(\mathrm{SD}=0.11)$ and the lowest $0.54(\mathrm{SD}=0.10)$. After 50 years of genetic rescue, the highest retention probability at any translocation rate was $0.21(\mathrm{SD}=0.05)$. For locally unique alleles with starting frequencies $<10 \%$ (Figure $6 \mathrm{~b}$ ), the highest mean retention probability at 10 years of genetic rescue was much lower at 
$0.20(\mathrm{SD}=0.16)$, with the lowest at $0.15(\mathrm{SD}=0.12)$. The highest retention probability at 50 years was $0.04(\mathrm{SD}=0.04)$.
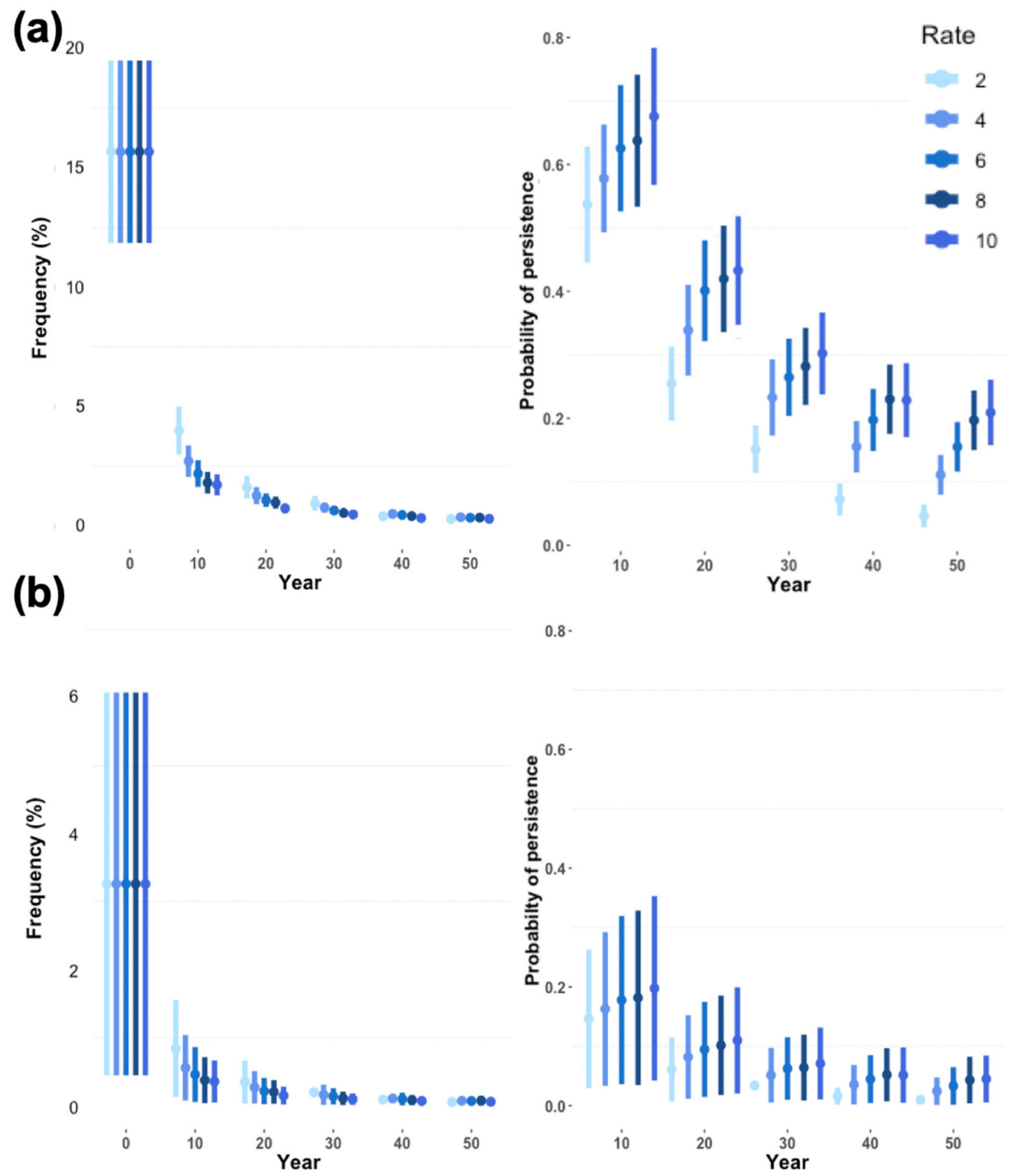

Figure 6. Mean frequencies $\pm 1 \mathrm{SD}$ and the probability of persistence $\pm 1 \mathrm{SD}$ of locally unique alleles with starting frequencies of $>10 \%$ (a) and $<10 \%$ (b) in the lowland population. Simulations began with 20 Yellingbo individuals, supplemented with different numbers of highland individuals per year (Rate). Frequency and probability of retention values were output every ten years, with values calculated from 200 replicate runs of each scenario. A carrying capacity of 1000 was specified.

Higher supplementation rates did not produce lower retention probabilities of locally unique alleles during genetic rescue but instead yielded overall higher probabilities (Figure 5). After 20 years of genetic rescue, retention probabilities were substantially higher when the translocation rate was increased from 8 to 10 individuals per year. The difference in retention probability at different translocation rates was statistically significant for all years: 10 years $(\mathrm{F}=169.02, \mathrm{df}=4, p<0.0001), 20$ years $(\mathrm{F}=238.2, \mathrm{df}=4, p<0.0001)$, 30 years $(\mathrm{F}=221.8, \mathrm{df}=4, p<0.0001), 40$ years $(\mathrm{F}=312.75, \mathrm{df}=4, p<0.0001)$, and 50 years $(\mathrm{F}=393.9$, $\mathrm{df}=4, p<0.0001)$. Differences between the translocation rates were also statistically significant for alleles with starting frequencies of $<10 \%$ : 10 years $(\mathrm{F}=64.52, \mathrm{df}=4, p<0.0001$ ), 
20 years $(\mathrm{F}=212.76, \mathrm{df}=4, p<0.0001), 30$ years $(\mathrm{F}=115.78, \mathrm{df}=4, p<0.0001), 40$ years $(\mathrm{F}=157.18, \mathrm{df}=4, p<0.0001)$, and 50 years $(\mathrm{F}=179.6, \mathrm{df}=4, p<0.0001)$.

Despite higher retention probabilities, locally unique alleles were lower in frequency at higher rates of gene flow (Figure 6). After 10 years of genetic rescue, the highest mean frequency for alleles with a $>10 \%$ starting frequency was $4.0 \%$ (SD $=1.0$ ), which was observed at the lowest supplementation rate of two individuals per year. Using a rate of 10 individuals per year yielded a substantially lower mean frequency of $1.6 \%(\mathrm{SD}=1.6)$. For alleles starting at $<10 \%$ frequency, the highest mean frequency was $0.8 \%(\mathrm{SD}=0.7)$, which was also observed at the lowest supplementation rate of the individuals per year. Using a rate of 10 individuals per year yielded a somewhat lower mean frequency of $0.4 \%$ $(\mathrm{SD}=0.3)$. The difference in allele frequency at different translocation rates was statistically significant for all years: 10 years $(\mathrm{F}=551.21, \mathrm{df}=4, p<0.001), 20$ years $(\mathrm{F}=268.1, \mathrm{df}=4$, $p<0.0001)$, 30 years $(\mathrm{F}=143.72, \mathrm{df}=4, p<0.0001), 40$ years, $(\mathrm{F}=30.5, \mathrm{df}=4, p<0.0001)$, and 50 years $(\mathrm{F}=8.9, \mathrm{df}=4, p<0.0001)$. Differences between the translocation rates were also statistically significant for the initially rare alleles $(<10 \%)$ : 10 years $(\mathrm{F}=64.52$, $\mathrm{df}=4$, $p<0.001), 20$ years $(\mathrm{F}=105.2, \mathrm{df}=4, p<0.0001), 30$ years $(\mathrm{F}=35.35, \mathrm{df}=4, p<0.0001)$, 40 years, $(\mathrm{F}=8.43, \mathrm{df}=4, p<0.0001)$, and 50 years $(\mathrm{F}=12.31, \mathrm{df}=4, p<0.0001)$.

\section{Discussion}

Many threatened wildlife species have highly disjunct distributions with little to no natural gene flow between populations, and translocations are critical for their long-term demographic and genetic stability [21]. Reintroducing populations within the historical range of a species or establishing populations beyond the historical range in low-threat areas (such as predator-free islands or reserves) are increasingly common [30,56,57]. Using predictive models to guide the founding of isolated populations with ongoing translocations is critical to maximising species-wide genetic variation [58]. We demonstrated how PVAs that incorporate inbreeding and inbreeding depression can predict how much locally unique variation can be retained while genetic rescue increases overall genetic variation.

PVAs predicted a very high probability that the lowland population of Leadbeater's possum will go extinct within two decades (100\% in 23 years) without any intervention. As environmental impacts on mortality and reproduction were minimised in simulations to make inferences based primarily upon genetics, this is likely an overestimate of time to extinction. Inbreeding depression strongly increased population decline in simulations, although a low carrying capacity was shown to curtail population size, even in the absence of inbreeding depression. This highlights the importance of providing lowland individuals with high-quality habitat to achieve population growth under genetic rescue. Reinforcing the population with only Yellingbo individuals (demographic rescue) did not sufficiently support population growth to reach our target size of 110 individuals, even using the largest translocation rate of 10 individuals per year. We demonstrated that a modest portion of neutral locally unique variation detected in the lowland population can likely be preserved through a combination of population expansion and outcrossing. Approximately $26 \%$ of unique lowland alleles occurred at frequencies above $10 \%$, which had a $>50 \%$ probability of persistence after 10 years of rescue.

There were several key assumptions in our PVAs that could bias estimates of genetic rescue and allele retention. Random mating among highland and lowland individuals was assumed in all scenarios. Preferential mating among individuals originating from similar habitat or populations can impede outcrossing attempts [59]. For Leadbeater's possum, this could cause translocated highland individuals to increase in abundance and supplant lowland individuals, increasing the risk of genetic swamping. This may be prevented by the careful choice of translocation sites, maximising the probability of highland-lowland pairings. Assortative mating among lowland individuals could also subvert genetic rescue efforts. However, females of some species become more selective in their mate choice if they are inbred, developing a preference for outbred males [60]. For lowland possums, this would result in a preference for highland mates, potentially 
increasing admixture rates and improving locally unique allele retention rates by increasing the fitness of individuals carrying such alleles, as well as increasing population growth and thus, reducing genetic drift. Our PVA models also assumed that the currently observed unique genetic variation is selectively neutral. If locally unique alleles are favourable, selection will increase their likelihood of persistence and frequency more so than if they are neutral. The effect of selection is expected to increase over time with increasing population size [22]. Alternatively, lowland-specific genetic variation may be maladapted if lowland individuals are relocated to a habitat that differs from Yellingbo, in which case, this variation would be selected against. All alleles were also assumed to be unlinked, whereas linkage can promote allele retention by genetic hitch-hiking of neutral alleles linked to loci under selection [31].

PVAs predicted that the supplementation rates of up to 10 individuals per year for 8 years will likely be necessary to meet population recovery targets. Although this supplementation rate is substantial, lower rates and less frequent supplements will likely be sufficient in reality to achieve the target population size. This is because the demographic rates modelled were estimated from data collected from the Yellingbo population during the 1990s, at which time the population was already inbred, having roughly half the genetic diversity of highland populations [36]. Therefore, the positive effects of genetic rescue and immigration will likely be greater than those estimated in this study, and fewer translocations might be necessary to provide the desired population benefits. Furthermore, Vortex does not account for a fixed genetic load in recipient populations [41], which means that fitness could be further improved by increasing heterozygosity at loci with fixed (100\% homozygous) deleterious alleles. Using assumptions similarly optimistic as ours, such as completely unrelated migrants, population growth projections were still underestimated by Vortex relative to the real data for translocations in another marsupial species (Bettongia penicillata ogilbyi) [61]. Thus, it is conceivable that the required rate of translocation estimated in our study is an overestimate. Conversely, a higher rate of supplementation may be necessary given that all individuals in our simulations were assumed to have the same mortality rate as local individuals. This is unrealistic, given that elevated post-translocation mortality is common in wildlife [62]. Genetic rescue should be implemented within an adaptive management framework, with post-translocation monitoring data incorporated into the models to make updated predictions $[63,64]$. Ongoing monitoring of fitness will also enable incorporation into future models of unlikely but possible effects of outbreeding depression and maladaptation. The genetic diversity and viability of source populations must also be considered, and further PVAs can help predict and mitigate the negative impacts of harvest [58,65].

The sensitivity of our PVAs to different parameter estimates suggests that management interventions that improve survival and reproductive output could facilitate more rapid population growth during genetic rescue. In particular, the proportion of adult females that were breeding and adult mortality had somewhat larger effects on growth rates than other parameters. Establishing colonies with translocated individuals and unpaired local possums could increase the proportion of females breeding in any given year. Adult mortality could be decreased by feral predator elimination measures, given that predation by feral cats and foxes is a known cause of mortality in Leadbeater's possum [66], and a common cause of translocation failure in Australian mammals [67]. Such measures could reduce the amount of supplementation required to reach a target population size.

Lower retention of locally unique lowland alleles at higher rates of supplementation with highland individuals was not observed in any of the simulated scenarios. However, locally unique alleles did decrease in frequency at higher supplementation rates, which is likely an effect of higher proportions of immigrants diluting neutral allele frequencies. A higher retention probability under more intensive gene flow was presumably caused by weaker genetic drift for scenarios where the population size increased more quickly. Thus, under the conditions used in simulations for the lowland population of Leadbeater's possum, genetic drift poses a greater threat to the preservation of unique genetic variation 
than dilution at higher rates of translocation. The number of lowland and highland founders will be a strong determinant of the number of alleles retained. Based on allele retention models in another translocated marsupial, using 20 founders is expected to retain $\sim 60 \%$ of rare (frequency $=0.05$ ) alleles and using at least 60 founders is expected to retain $\sim 90 \%$ of rare alleles [30]. Minimising genetic and demographic stochasticity in small populations by rapid supplementation has been recommended in other species to maximise the effectiveness of population reinforcement [68]. Our study suggests that this principle could also apply to genetic rescue and the preservation of locally unique variation. Although the frequency of these locally unique alleles may be reduced at higher supplementation rates, this would be offset by the benefits of a larger population size (such as reduced stochasticity), and selection will increase their frequency if they present a fitness advantage.

Previous experimental investigations into genetic swamping effects following genetic rescue have focused on locally adaptive alleles $[22,69]$. However, genetic variation that is not currently subject to selection (such as cryptic variation that only alters phenotype under atypical circumstances) can become useful if environments change; such enhancement of evolutionary potential would preserve apparently neutral variation in the face of uncertain futures $[14,70]$. Although only a small proportion of alleles $(0.55-2.2 \%$ of loci) were unique to the lowland population at Yellingbo and none were fixed, it is possible that they could contribute to local adaptation or evolutionary potential. Much unique variation will have been lost from the lowland population owing to the small population size over decades, with an observable reduction in the number of locally unique alleles from 2011 to 2019 (Supplementary Figure S4). Nonetheless, locally adaptive genetic variation can persist in the presence of strong genetic drift [71]. The detection of locally unique variation is also dependent on marker type and density, with SNPs representing only one form of genetic variation. Other types of variation that contribute to local adaptation include structural and copy number variants [72]. Such variation is unlikely represented in SNP datasets, making the amount of unique variation among populations an underestimate when using SNPs alone. Deleterious alleles that are recent mutations tend to be prevalent at low frequencies [73], and many locally unique alleles detected were very rare (17\% with $<1 \%$ frequency). However, it is unclear whether the locally unique alleles detected could be deleterious, because intense genetic drift in the lowland population will have strongly influenced allele frequencies.

The contribution of ongoing post-rescue gene flow to demographic and genetic stability is another important management consideration. Although there is some potential that supplementing the lowland population with individuals from large populations will increase the genetic load, maintaining heterozygosity and minimising inbreeding via occasional gene flow will mask recessive genetic load. Some studies have cited pronounced population declines following the immigration of as few as a single individual as evidence that genetic rescue can further imperil small populations by introducing deleterious alleles that are more numerous in larger populations [74]. Fitness decline with insufficient genetic rescue is unsurprising because inbreeding (identity-by-descent) inevitably increases to pretranslocation levels in very small populations without ongoing gene flow [75]. Founding a new lowland population near existing Central Highlands populations could enable dispersal between populations, which would increase long-term population viability. Natural dispersal could also facilitate the spread of lowland genetic variation that is potentially adaptive to warmer conditions, improving the fitness of Central Highlands populations under climate change. The establishment of self-sustaining populations is a highly soughtafter goal in conservation management [76]. However, populations that are genetically isolated with $N_{e}<1000$ will inevitably require at least occasional assisted gene flow if natural gene flow is not possible $[1,58]$. Early interventions that prevent extreme reductions in $N_{e}$ and mitigating the underlying causes of a small population size are the best means of reducing dependency on conservation management. 


\section{Conclusions}

We estimate that without intervention, all unique lowland Leadbeater's possum genetic variation will be lost due to extinction of the last population of the lineage within approximately two decades, whereas modest amounts of this unique variation can be preserved with genetic rescue. A higher proportion of locally unique variation is predicted to be retained by adding a greater number of highland animals each year with genetic rescue of the lowland population. Ongoing gene flow from highland populations will also be critical to maintaining long-term demographic and genetic stability within the lowland population. Although PVAs are based on many assumptions, they can form an integral component of adaptive genetic management and offer flexibility to incorporate post-translocation data to resolve uncertainties such as relative fitness among outbred and inbred individuals.

Supplementary Materials: The following are available online at https:/ /www.mdpi.com/article/10.3 $390 /$ d13080382/s1, Figure S1. Population growth using values of mortality at $0-1$ years estimated in the 1990's and using estimated values $+6 \%$ to produce a demographically stable population in the absence of inbreeding depression. Figure S2. Effect of percent inbreeding depression due to lethal recessive alleles (as opposed to heterozygote advantage) on genetic rescue. Effects are shown as change in population size $(\mathrm{N})$ with genetic rescue by supplementing Yellingbo founders with two highland individuals per year. Percentages of 0-75 inbreeding due to lethal recessives produced comparable changes in population size. Inbreeding depression $100 \%$ due to lethals produced much more positive population growth. Figure S3. Frequencies of locally-unique alleles at Yellingbo from 2011-2019, and 2019 alone. Figure S4. PVA input parameter sensitivity tests. Mean growth rate (r) from 200 replicate runs of 50-year simulations. All scenarios began with 110 individuals, excluded inbreeding depression, and used a carrying capacity of 1,0000. A baseline scenario was run using actual input parameter values (except with $0-1$ yr mortality increased by $6 \%$ ), which gave a stable population of approximately 110 individuals. Output values for this baseline scenario are shown by the dashed line. For each scenario shown on the x-axis, a single input parameter was either increased (Low) or increased (High) by $10 \%$ of its baseline value to determine its relative influence on simulation outcomes.

Author Contributions: Conceptualization, J.P.Z. and P.S.; methodology, J.P.Z., D.H., A.P. and P.S.; software, J.P.Z.; validation, J.P.Z., D.H., A.P. and P.S.; formal analysis, J.P.Z.; investigation, J.P.Z.; resources, P.S. and J.P.Z.; data curation, J.P.Z.; writing—original draft preparation, J.P.Z.; writingreview and editing, J.P.Z., D.H., A.P. and P.S.; visualization, J.P.Z.; supervision, P.S and A.P.; project administration, J.P.Z. and P.S.; funding acquisition, P.S. and J.P.Z. All authors have read and agreed to the published version of the manuscript.

Funding: This research was funded by an ARC Linkage Grant LP160100482 with Partner Organizations Victorian Department of Environment, Land, Water, and Planning (DELWP), Diversity Arrays Technology, Zoos Victoria, Australian Capital Territory Environment, Planning \& Sustainable Development Directorate, University of Canberra, and Western Australian Department of Biodiversity, Conservation and Attractions. Joanne Antrobus from Parks Victoria has provided substantial support to the long-term monitoring program for Leadbeater's possum at Yellingbo. J.P.Z. and D.H. were supported by Monash University internal funds and Research and Training Program Scholarships (formerly Australian Postgraduate Award), and awards from the Holsworth Wildlife Research Endowment. This research drew on materials from an Australian Academy of Sciences Research Award for the Conservation of Endangered Australian Vertebrate Species to Andrea Taylor.

Institutional Review Board Statement: All population monitoring and tissue sample collection procedures were approved by the Zoos Victoria Animal Ethics Committee (project ZV14004 approved 16/04/2014 and project ZV18001 approved 15/03/2018).

Informed Consent Statement: Not applicable.

Data Availability Statement: Supporting data for this publication are publicly available at the Monash University online data repository (https:/ / doi.org/10.26180/15019797, accessed on 12 August 2021). 
Acknowledgments: We wish to thank Bob Lacy for extremely useful advice regarding PVAs. We thank the editors and Diversity for the invitation to submit a manuscript for consideration in this special issue, three anonymous reviewers for their comments, and Carolyn Hogg and Steve Cooper who assessed an earlier version.

Conflicts of Interest: The authors declare no conflict of interest.

\section{References}

1. Frankham, R.; Ballou, J.D.; Ralls, K.; Eldridge, M.D.B.; Dudash, M.R.; Fenster, C.B.; Lacy, R.C.; Sunnucks, P. Genetic Management of Fragmented Animal and Plant Populations; Oxford University Press: Oxford, UK, 2017.

2. Whiteley, A.R.; Fitzpatrick, S.W.; Funk, W.C.; Tallmon, D.A. Genetic Rescue to the Rescue. Trends Ecol. Evol. 2015, 30, 42-49. [CrossRef] [PubMed]

3. Hedrick, P.W.; Garcia-Dorado, A. Understanding Inbreeding Depression, Purging, and Genetic Rescue. Trends Ecol. Evol. 2016, 31, 940-952. [CrossRef]

4. Hedrick, P.W.; Fredrickson, R. Genetic Rescue Guidelines with Examples from Mexican Wolves and Florida Panthers. Conserv. Genet. 2010, 11, 615-626. [CrossRef]

5. Eizaguirre, C.; Baltazar-Soares, M. Evolutionary Conservation-Evaluating the Adaptive Potential of Species. Evol. Appl. 2014, 7, 963-967. [CrossRef]

6. Fagan, W.F.; Holmes, E.E. Quantifying the Extinction Vortex. Ecol. Lett. 2006, 9, 51-60. [CrossRef] [PubMed]

7. Blomqvist, D.; Pauliny, A.; Larsson, M.; Flodin, L.-A. Trapped in the Extinction Vortex? Strong Genetic Effects in a Declining Vertebrate Population. BMC Evol. Biol. 2010, 10, 33. [CrossRef]

8. vander Wal, E.; Garant, D.; Calmé, S.; Chapman, C.A.; Festa-Bianchet, M.; Millien, V.; Rioux-Paquette, S.; Pelletier, F. Applying Evolutionary Concepts to Wildlife Disease Ecology and Management. Evol. Appl. 2014, 7, 856-868. [CrossRef] [PubMed]

9. Hoffmann, A.A.; Miller, A.D.; Weeks, A.R. Genetic Mixing for Population Management: From Genetic Rescue to Provenancing. Evol. Appl. 2021, 14, 634-652. [CrossRef] [PubMed]

10. Frankham, R. Genetic Rescue of Small Inbred Populations: Meta-Analysis Reveals Large and Consistent Benefits of Gene Flow. Mol. Ecol. 2015, 24, 2610-2618. [CrossRef] [PubMed]

11. Love Stowell, S.M.; Pinzone, C.A.; Martin, A.P. Overcoming Barriers to Active Interventions for Genetic Diversity. Biodivers. Conserv. 2017, 26, 1753-1765. [CrossRef]

12. Odell, E.A.; Heffelfinger, J.R.; Rosenstock, S.S.; Bishop, C.J.; Liley, S.; González-Bernal, A.; Velasco, J.A.; Martínez-Meyer, E. Perils of Recovering the Mexican Wolf Outside of Its Historical Range. Biol. Conserv. 2018, 220, 290-298. [CrossRef]

13. Bell, D.A.; Robinson, Z.L.; Funk, W.C.; Fitzpatrick, S.W.; Allendorf, F.W.; Tallmon, D.A.; Whiteley, A.R. The Exciting Potential and Remaining Uncertainties of Genetic Rescue. Trends Ecol. Evol. 2019, 34, 1070-1079. [CrossRef]

14. Harrisson, K.A.; Pavlova, A.; Telonis-Scott, M.; Sunnucks, P. Using Genomics to Characterize Evolutionary Potential for Conservation of Wild Populations. Evol. Appl. 2014, 7, 1008-1025. [CrossRef]

15. Roberts, D.G.; Gray, C.A.; West, R.J.; Ayre, D.J. Marine Genetic Swamping: Hybrids Replace an Obligately Estuarine Fish. Mol. Ecol. 2010, 19, 508-520. [CrossRef]

16. Hedrick, P.W. Gene Flow and Genetic Restoration: The Florida Panther as a Case Study. Conserv. Biol. 1995, 9, 996-1007. [CrossRef] [PubMed]

17. Liddell, E.; Sunnucks, P.; Cook, C.N. To Mix or Not to Mix Gene Pools for Threatened Species Management? Few Studies Use Genetic Data to Examine the Risks of Both Actions, but Failing to Do so Leads Disproportionately to Recommendations for Separate Management. Biol. Conserv. 2021, 256, 109072. [CrossRef]

18. Aitken, S.N.; Whitlock, M.C. Assisted Gene Flow to Facilitate Local Adaptation to Climate Change. Annu. Rev. Ecol. Evol. Syst. 2013, 44, 367-388. [CrossRef]

19. Sjöstrand, A.E.; Sjödin, P.; Jakobsson, M. Private Haplotypes Can Reveal Local Adaptation. BMC Genet. 2014, 15, 61. [CrossRef] [PubMed]

20. Thrimawithana, A.H.; Ortiz-Catedral, L.; Rodrigo, A.; Hauber, M.E. Reduced Total Genetic Diversity Following Translocations? A Metapopulation Approach. Conserv. Genet. 2013, 14, 1043-1055. [CrossRef]

21. Weeks, A.R.; Stoklosa, J.; Hoffmann, A.A. Conservation of Genetic Uniqueness of Populations May Increase Extinction Likelihood of Endangered Species: The Case of Australian Mammals. Front. Zool. 2016, 13, 31. [CrossRef]

22. Fitzpatrick, S.W.; Bradburd, G.S.; Kremer, C.T.; Salerno, P.E.; Angeloni, L.M.; Funk, W.C. Genomic and Fitness Consequences of Genetic Rescue in Wild Populations. Curr. Biol. 2020, 30, 517-522.e5. [CrossRef]

23. Willi, Y.; Griffin, P.; van Buskirk, J. Drift Load in Populations of Small Size and Low Density. Heredity 2013, 110, $296-302$. [CrossRef]

24. Fernandez-Fournier, P.; Lewthwaite, J.M.M.; Mooers, A.Ø. Do We Need to Identify Adaptive Genetic Variation When Prioritizing Populations for Conservation? Conserv. Genet. 2021, 22, 205-216. [CrossRef]

25. Ørsted, M.; Hoffmann, A.A.; Sverrisdóttir, E.; Nielsen, K.L.; Kristensen, T.N. Genomic Variation Predicts Adaptive Evolutionary Responses Better than Population Bottleneck History. PLoS Genet. 2019, 15, e1008205. [CrossRef] [PubMed]

26. Hoban, S.; Bertorelle, G.; Gaggiotti, O.E. Computer Simulations: Tools for Population and Evolutionary Genetics. Nat. Rev. Genet. 2012, 13, 110-122. [CrossRef] 
27. Pavlova, A.; Beheregaray, L.B.; Coleman, R.; Gilligan, D.; Harrisson, K.A.; Ingram, B.A.; Kearns, J.; Lamb, A.M.; Lintermans, M.; Lyon, J.; et al. Severe Consequences of Habitat Fragmentation on Genetic Diversity of an Endangered Australian Freshwater Fish: A Call for Assisted Gene Flow. Evol. Appl. 2017, 10, 531-550. [CrossRef]

28. Reynolds, M.H.; Weiser, E.; Jamieson, I.; Hatfield, J.S. Demographic Variation, Reintroduction, and Persistence of an Island Duck (Anas Laysanensis). J. Wildl. Manag. 2013, 77, 1094-1103. [CrossRef]

29. Schueller, A.M.; Hayes, D.B. Minimum Viable Population Size for Lake Sturgeon (Acipenser Fulvescens) Using an IndividualBased Model of Demographics and Genetics. Can. J. Fish. Aquat. Sci. 2010, 68, 62-73. [CrossRef]

30. White, D.J.; Ottewell, K.; Spencer, P.B.S.; Smith, M.; Short, J.; Sims, C.; Mitchell, N.J. Genetic Consequences of Multiple Translocations of the Banded Hare-Wallaby in Western Australia. Diversity 2020, 12, 448. [CrossRef]

31. Harris, K.; Zhang, Y.; Nielsen, R. Genetic Rescue and the Maintenance of Native Ancestry. Conserv. Genet. 2019, 20, 59-64. [CrossRef]

32. Greet, J.; Harley, D.; Ashman, K.; Watchorn, D.; Duncan, D. The Vegetation Structure and Condition of Contracting Lowland Habitat for Leadbeater's Possum (Gymnobelideus Leadbeateri). Aust. Mammal. 2020. First published online 16 December 2020. [CrossRef]

33. Harley, D. An Overview of Actions to Conserve Leadbeater's Possum “Gymnobelideus Leadbeateri”. Vic. Nat. 2016, 133, 85-97. [CrossRef]

34. Harley, D.; Worley, M.; Harley, T. The Distribution and Abundance of Leadbeater's Possum Gymnobelideus Leadbeateri in Lowland Swamp Forest at Yellingbo Nature Conservation Reserve. Aust. Mammal. 2005, 27, 7-15. [CrossRef]

35. Hansen, B.D.; Taylor, A.C. Isolated Remnant or Recent Introduction? Estimating the Provenance of Yellingbo Leadbeater's Possums by Genetic Analysis and Bottleneck Simulation. Mol. Ecol. 2008, 17, 4039-4052. [CrossRef]

36. Hansen, B.D.; Harley, D.K.P.; Lindenmayer, D.B.; Taylor, A.C. Population Genetic Analysis Reveals a Long-Term Decline of a Threatened Endemic Australian Marsupial. Mol. Ecol. 2009, 18, 3346-3362. [CrossRef]

37. Zilko, J.P.; Harley, D.; Hansen, B.; Pavlova, A.; Sunnucks, P. Accounting for Cryptic Population Substructure Enhances Detection of Inbreeding Depression with Genomic Inbreeding Coefficients: An Example from a Critically Endangered Marsupial. Mol. Ecol. 2020, 29, 2978-2993. [CrossRef]

38. Harley, D. The Application of Zoos Victoria's "Fighting Extinction" Commitment to the Conservation of Leadbeater's Possum "Gymnobelideus Leadbeateri". Vic. Nat. 2012, 129, 175-180. [CrossRef]

39. Harley, D.K.P.; Lill, A. Reproduction in a Population of the Endangered Leadbeater's Possum Inhabiting Lowland Swamp Forest. J. Zool. 2007, 272, 451-457. [CrossRef]

40. Lacy, R.C. Lessons from 30 Years of Population Viability Analysis of Wildlife Populations. Zoo Biol. 2019, 38, 67-77. [CrossRef]

41. Lacy, R.C. VORTEX: A Computer Simulation Model for Population Viability Analysis. Wildl. Res. 1993, 20, 45-65. [CrossRef]

42. O'Grady, J.J.; Brook, B.W.; Reed, D.H.; Ballou, J.D.; Tonkyn, D.W.; Frankham, R. Realistic Levels of Inbreeding Depression Strongly Affect Extinction Risk in Wild Populations. Biol. Conserv. 2006, 133, 42-51. [CrossRef]

43. Simmons, M.J.; Crow, J.F. Mutations Affecting Fitness in Drosophila Populations. Annu. Rev. Genet. 1977, 11, 49-78. [CrossRef] [PubMed]

44. Harley, D.K.P. The Life History and Conservation of Leadbeater's Possum (Gymnobelideus leadbeateri) in Lowland Swamp Forest. PhD Thesis, Monash University, Melbourne, Australia, 2005.

45. Charlesworth, D.; Willis, J.H. The Genetics of Inbreeding Depression. Nat. Reviews. Genet. 2009, 10, 783-796. [CrossRef] [PubMed]

46. Sunnucks, P.; Hales, D.F. Numerous Transposed Sequences of Mitochondrial Cytochrome Oxidase I-II in Aphids of the Genus Sitobion (Hemiptera: Aphididae). Mol. Biol. Evol. 1996, 13, 510-524. [CrossRef]

47. Kilian, A.; Wenzl, P.; Huttner, E.; Carling, J.; Xia, L.; Blois, H.; Caig, V.; Heller-Uszynska, K.; Jaccoud, D.; Hopper, C.; et al. Diversity Arrays Technology: A Generic Genome Profiling Technology on Open Platforms. In Methods in Molecular Biology; Humana Press: Clifton, NJ, USA, 2012; Volume 888, pp. 67-89.

48. Gruber, B.; Unmack, P.J.; Berry, O.F.; Georges, A. Dartr: An r Package to Facilitate Analysis of SNP Data Generated from Reduced Representation Genome Sequencing. Mol. Ecol. Resour. 2018, 18, 691-699. [CrossRef]

49. Kamvar, Z.N.; Tabima, J.F.; Grünwald, N.J. Poppr: An R Package for Genetic Analysis of Populations with Clonal, Partially Clonal, and/or Sexual Reproduction. PeerJ 2014, 2, e281. [CrossRef]

50. Thompson, E.A. Population Correlation and Population Kinship. Theor. Popul. Biol. 1976, 10, 205-226. [CrossRef]

51. Städele, V.; Vigilant, L. Strategies for Determining Kinship in Wild Populations Using Genetic Data. Ecol. Evol. 2016, 6, 6107-6120. [CrossRef]

52. Goudet, J. Hierfstat, a Package for $\mathrm{r}$ to Compute and Test Hierarchical F-Statistics. Mol. Ecol. Notes 2005, 5, 184-186. [CrossRef]

53. Goudet, J.; Kay, T.; Weir, B.S. How to Estimate Kinship. Mol. Ecol. 2018, 27, 4121-4135. [CrossRef] [PubMed]

54. Weir, B.S.; Goudet, J. A Unified Characterization of Population Structure and Relatedness. Genetics 2017, 206, 2085. [CrossRef] [PubMed]

55. Wobbrock, J.O.; Findlater, L.; Gergle, D.; Higgins, J.J. The Aligned Rank Transform for Nonparametric Factorial Analyses Using Only Anova Procedures. In Proceedings of the SIGCHI Conference on Human Factors in Computing Systems; Association for Computing Machinery, New York, NY, USA, 12 May 2011; pp. 143-146.

56. Ryan, C.M.; Hobbs, R.J.; Valentine, L.E. Bioturbation by a Reintroduced Digging Mammal Reduces Fuel Loads in an Urban Reserve. Ecol. Appl. 2020, 30, e02018. [CrossRef] [PubMed] 
57. Burbidge, A.A.; Abbott, I. Mammals on Western Australian Islands: Occurrence and Preliminary Analysis. Aust. J. Zool. 2017, 65, 183-195. [CrossRef]

58. Pacioni, C.; Wayne, A.F.; Page, M. Guidelines for Genetic Management in Mammal Translocation Programs. Biol. Conserv. 2019, 237, 105-113. [CrossRef]

59. Slade, B.; Parrott, M.L.; Paproth, A.; Magrath, M.J.L.; Gillespie, G.R.; Jessop, T.S. Assortative Mating among Animals of Captive and Wild Origin Following Experimental Conservation Releases. Biol. Lett. 2014, 10, 20140656. [CrossRef] [PubMed]

60. Pilakouta, N.; Smiseth, P.T. Female Mating Preferences for Outbred versus Inbred Males Are Conditional upon the Female's Own Inbreeding Status. Anim. Behav. 2017, 123, 369-374. [CrossRef]

61. Pacioni, C.; Atkinson, A.; Trocini, S.; Rafferty, C.; Morley, K.; Spencer, P.B.S. Is Supplementation an Efficient Management Action to Increase Genetic Diversity in Translocated Populations? Ecol. Manag. Restor. 2020, 21, 123-130. [CrossRef]

62. Armstrong, D.P.; Seddon, P.J. Directions in Reintroduction Biology. Trends Ecol. Evol. 2008, 23, 20-25. [CrossRef]

63. Westgate, M.J.; Likens, G.E.; Lindenmayer, D.B. Adaptive Management of Biological Systems: A Review. Biol. Conserv. 2013, 158, 128-139. [CrossRef]

64. Pacioni, C.; Trocini, S.; Wayne, A.F.; Rafferty, C.; Page, M. Integrating Population Genetics in an Adaptive Management Framework to Inform Management Strategies. Biodivers. Conserv. 2020, 29, 947-966. [CrossRef]

65. Verdon, S.J.; Mitchell, W.F.; Clarke, M.F. Can Flexible Timing of Harvest for Translocation Reduce the Impact on Fluctuating Source Populations? Wildl. Res. 2021, 28, 458-469. [CrossRef]

66. McComb, L.B.; Lentini, P.E.; Harley, D.K.P.; Lumsden, L.F.; Antrobus, J.S.; Eyre, A.C.; Briscoe, N.J. Feral Cat Predation on Leadbeater's Possum (Gymnobelideus Leadbeateri) and Observations of Arboreal Hunting at Nest Boxes. Aust. Mammal. 2019, 41, 262-265. [CrossRef]

67. Pacioni, C.; Williams, M.R.; Lacy, R.C.; Spencer, P.B.S.; Wayne, A.F. Predators and Genetic Fitness: Key Threatening Factors for the Conservation of a Bettong Species. Pac. Conserv. Biol. 2017, 23, 200-212. [CrossRef]

68. Hardy, M.A.; Hull, S.D.; Zuckerberg, B. Swift Action Increases the Success of Population Reinforcement for a Declining Prairie Grouse. Ecol. Evol. 2018, 8, 1906-1917. [CrossRef] [PubMed]

69. Poirier, M.-A.; Coltman, D.W.; Pelletier, F.; Jorgenson, J.; Festa-Bianchet, M. Genetic Decline, Restoration and Rescue of an Isolated Ungulate Population. Evol. Appl. 2019, 12, 1318-1328. [CrossRef]

70. Zheng, J.; Payne, J.L.; Wagner, A. Cryptic Genetic Variation Accelerates Evolution by Opening Access to Diverse Adaptive Peaks. Science 2019, 365, 347. [CrossRef] [PubMed]

71. Funk, W.C.; Lovich, R.E.; Hohenlohe, P.A.; Hofman, C.A.; Morrison, S.A.; Sillett, T.S.; Ghalambor, C.K.; Maldonado, J.E.; Rick, T.C.; Day, M.D.; et al. Adaptive Divergence despite Strong Genetic Drift: Genomic Analysis of the Evolutionary Mechanisms Causing Genetic Differentiation in the Island Fox (Urocyon Littoralis). Mol. Ecol. 2016, 25, 2176-2194. [CrossRef]

72. Tigano, A.; Friesen, V.L. Genomics of Local Adaptation with Gene Flow. Mol. Ecol. 2016, 25, 2144-2164. [CrossRef] [PubMed]

73. Bosse, M.; Megens, H.-J.; Derks, M.F.L.; de Cara, Á.M.R.; Groenen, M.A.M. Deleterious Alleles in the Context of Domestication, Inbreeding, and Selection. Evol. Appl. 2018, 12, 6-17. [CrossRef] [PubMed]

74. Kyriazis, C.C.; Wayne, R.K.; Lohmueller, K.E. Strongly Deleterious Mutations Are a Primary Determinant of Extinction Risk Due to Inbreeding Depression. Evol. Lett. 2021, 5, 33-47. [CrossRef]

75. Ralls, K.; Sunnucks, P.; Lacy, R.C.; Frankham, R. Genetic Rescue: A Critique of the Evidence Supports Maximizing Genetic Diversity Rather than Minimizing the Introduction of Putatively Harmful Genetic Variation. Biol. Conserv. 2020, $251,108784$. [CrossRef]

76. Mussmann, S.M.; Douglas, M.R.; Anthonysamy, W.J.B.; Davis, M.A.; Simpson, S.A.; Louis, W.; Douglas, M.E. Genetic Rescue, the Greater Prairie Chicken and the Problem of Conservation Reliance in the Anthropocene. R. Soc. Open Sci. 2021, $4,160736$. [CrossRef] 